\title{
Design of Intelligent Monitoring System Based on ZigBee Wireless Network
}

\author{
Wang Yexing ${ }^{1, a}$, Li Lin ${ }^{2, b}$ \\ ${ }^{1}$ Qiqihaer medical university, Qiqihaer, 161006, China \\ 2 Qigihar Center for Disease Control and Prevention, Qiqihaer, 161006, China \\ aemail: wangyexing789@126.com, bemail: qqhelilin@126.com
}

Keywords: wireless sensor network; intelligent monitoring; multi-parameter; ZigBee

\begin{abstract}
Current medical monitoring equipment has disadvantages of high power, high cost, fixed monitoring, more wiring, etc. In the paper, a set of intelligent medical monitoring system based on ZigBee wireless sensor network is developed aiming at these disadvantages. High-frequency wireless multi-channel data transmission mode is adopted for transferring information between medical sensor and monitoring control instruments. The product has advantages of low power, small size, low network operation cost, etc.
\end{abstract}

\section{Introduction}

Medical monitoring refers to testing and monitoring on human physiological and pathological states. It can be used for real-time continuous monitoring of vital life characteristic parameters of patients for long time. The parameters can be transmitted to doctor. Doctor can conduct corresponding diagnosis and treatment according to testing results. Currently, wired bedside monitoring equipment is mostly used as monitoring equipment in domestic hospitals. The monitoring equipment has disadvantages of inconvenient transportation, poor mobility, complicated wiring, etc. Patients suffer from high psychological pressure. Therefore the diagnosis results can be deviated from actual condition, thereby affecting early detection and treatment of diseases. Therefore, low-power and low-cost wireless medical monitoring system has become a hot topic of research in recent years with rapid development of wireless sensor network technology. ZigBee technology belongs to a short-range and low-power wireless communication technology, which uses $2.4 \mathrm{GHz}$ band. Frequency hopping and spread spectrum technology are adopted. The product has characteristics of low power consumption, low cost, low rate, low latency, high network capacity, etc $^{[1-2]}$. In the paper, a medical monitoring system based on ZigBee wireless sensor network is designed. Physiological information of the monitored object can be transmitted from sensor to monitoring master station by wireless multi-channel data transmission mode, thereby reducing wired connection between sensor and equipment in monitoring master station. The monitored object can move freely in certain scope, thereby guaranteeing accuracy of monitoring parameters.

\section{Network Technology Based on ZigBee Wireless Sensor}

Sensing, computing and network linking abilities are combined in wireless sensor network. Different sensors can be used for monitoring and detecting surrounding environment and states of specific objects in the sensing scope. The states can be transmitted to host machine by wireless network $^{[3]}$. When system management personnel receives the information, the information can be suitably treated by the technology. Wireless sensor network requires that the nodes should be provided with wireless communication ability. Currently, there are many mainstream wireless communication protocols ${ }^{[4]}$. The transmission protocols have own advantages characteristics. However, ZigBee protocol is mainly used in wireless sensor network technology. ZigBee is a new wireless network technology characterized by short range, low complexity, low power and low cost, which is mainly used for short-range wireless connection. It can realize communication by mutual coordination among many tiny sensors according to IEEE802.15.4 standard. The sensors only 
require little energy ${ }^{[5]}$. Data can be transmitted to another sensor from one sensor through wireless radio waves in the form of relay, therefore they have high communication efficiency. is very high. ZigBee technology will have the lowest power consumption and cost compared with various wireless communication technologies. ZigBee has starting point of realizing an easily-implemented low-cost wireless network. Battery can be adopted for power supply due to its low power consumption. The product can maintain operation for six months to several years. It is determined that ZigBee technology is suitable for carrying business with low data traffic due to its characteristics of low data rate and small communication rang. Various devices can be simply networked through ZigBee. It creates conditions for ubiquitous network in the future as a global standard.

\section{Design of Intelligent Monitoring System Structure}

In the monitoring system designed in the paper, nodes of wireless sensor are randomly distributed within network coverage area, which can be automatically added to wireless monitoring network. Data transmission can be realized through multi-hop relay mode. Mesh topology structure is adopted in the medical monitoring network design in the paper in order to conveniently expand equipment quantity and network effective area in monitoring network. The system constitutes a miniature monitoring network by monitoring base station equipment and ZigBee sensor nodes. Central controllers are used on sensor nodes for controlling the to-be-monitored vital sign sensors, thereby collecting data. Data can be transmitted to monitoring base station equipment through ZigBee wireless communication mode. Data can be transmitted to the connected PC or other network equipment through the base station devices. Data can be transmitted to remote medical monitoring center through Internet network. Professional medical personnel can conduct statistic observation on data, provide necessary advisory services, and realize remote medical series.

ZigBee technology supports three network topology structures, namely star network, cluster-tree networks and mesh networks. All terminal devices can communicate directly with coordinators in star network. Direct communication among terminal nodes is not allowed. The star network is suitable for occasions with small network coverage scope and few network devices. However, any two nodes in network coverage scope can realize direct communication in cluster-tree network and mesh network. There are generally three devices with different functions in ZigBee network, namely ZigBee coordinator, ZigBee router and ZigBee terminal device. \# coordinator is a full-function device, which acts as center of network, and is mainly responsible for establishing and starting network, configuring network parameters, etc. ZigBee router is a full-function device that acts as a relay among remote devices, thereby realizing data transfer of remote devices, and effectively expanding the coverage scope of network. Terminal devices may be FFD or a reduced function device RFD. It mainly acts as sensor node in network.

Emergency personnel in the ambulance also can realize real-time transmission of patient condition through GPRS, therefore hospital emergency can prepare immediately. Medical sensor nodes can be set according to different needs, therefore the system has great flexibility and scalability. Meanwhile, the system is connected to Internet, larger community medical monitoring network, hospital network and even medical monitoring network for the whole city and country can be formed.

\section{Design of Intelligent Monitoring System Hardware}

The hardware part of the system integrates sensors and communications modules. Testing sensor is responsible for collecting physiological information data of patients. Communication module is responsible for wireless communication. Transmitted data can be forwarded to monitoring host machine. Monitoring software of monitoring center is responsible for data analysis and display. System is connected with database. Long-term clinical data of patients can be saved. Meanwhile, acquisition of patient medical data is supported 


\section{Design of Sensor Node Hardware}

Sensor node is regarded as physiological parameter acquisition detection device, which can be used for collecting physiological parameters of monitored objects through various physiological sensors. Data can be stored to MCU temporary storage buffer area after passing though signal conditioning circuit, thereby waiting to be sent. Sensor node is mainly composed of physiological parameter sensor, microcontroller and wireless communication circuit. TI company's MSP430F149 is adopted as main controller for sensor nodes. MSP430 MCU has advantages of ultra-low power consumption, low radiation, high rate, etc., which is widely applied in various medical devices. Wireless communication circuit regards chipcon company's CC2430 microcontrollers as core. It can well support ZigBee protocol, which act as core for realizing wireless monitoring network. After master controller of sensor nodes collect physiological data of monitored objects in real time, data can be transferred to MSP430 wireless communication module, thereby realizing timing transmission of data. Sensor node hardware structure is shown in figure 1.

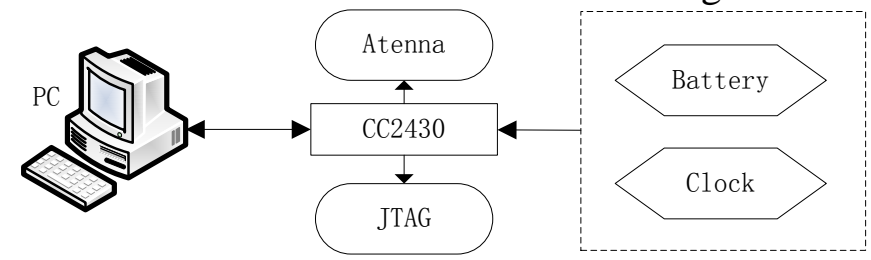

Fig.1. Sensor Node Hardware Structure

Medical wireless sensor node has main function of collecting human physiological indicator data, or condition or medical process condition of some medical devices can be dynamically monitored. Data is transmitted to equipment in monitoring base station through radio communication mode. Medical sensor node mainly includes four parts: physiological information and data acquisition unit, wireless data communication unit, processor unit and power supply. Processing unit is mainly divided into five parts: CPU, memory, AD converter, test tape and digital display. TI Company's MSP430 MCU is adopted according to demands of low power consumption and processing ability. The memory part is mainly used for storing temporary data collected by storage sensor. After data is transmitted by processor, much data is not stored in sensor node. ADC12 and DAC12 are used for converting analog signals transmitted from test tape. Medical sensor node block diagram and processor unit are shown in figure 2.

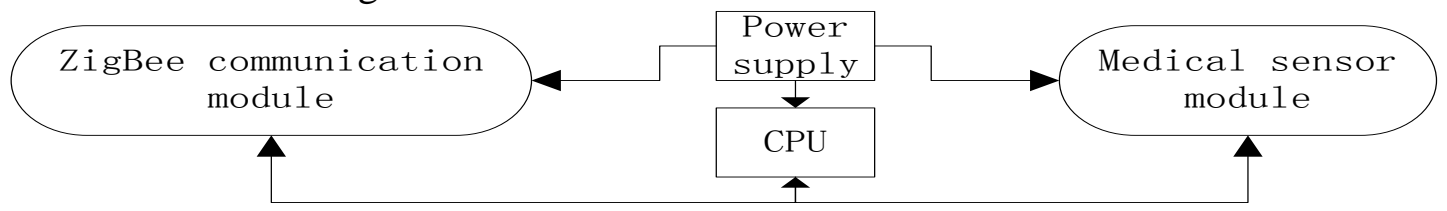

Fig.2. Medical Wireless Sensor Node Block Diagram

\section{Design of Communication Node Hardware}

Router node is regarded as relay device among monitoring network devices in monitoring network. Data in monitoring network can be transferred from sensor node to monitoring master station, thereby effectively expanding coverage scope of monitoring network. Router nodes can establish rational and effective data transmission routes for the physiological data of sensor node according to related information of some sensor node, therefore data can be transmitted from sensor node to monitoring master station in more efficient mode. The central node, network coordinator, is responsible for establishing wireless network, sending network beacons, storing network node information, selecting route for message, and other tasks. Although they are greatly different in function, their hardware composition is basically the same, mainly including processor, clock circuit, memory, level switching circuit, antenna, etc. Router node and central node are provided with CC2430 as core controllers. It can realize wireless communication function through a few peripheral circuits. 


\section{Design of Monitoring System Software}

System software design mainly belongs to software system design of monitoring center. Software system of monitoring center is the core to realize intelligent wireless monitoring system in ward. Main functions include transmission of command data to base station and sensor, including positioning instructions, request data instructions and configuration instructions. The system receives data from base station, including patient calls, vital sign data, configuration data, location data and command implementation return value. Patient vital sign data cared by the host machine is provided for medical monitoring host machine. It has functions of realizing sound and light alarm and voice. Meanwhile, vital sign data of all monitored points are recorded in the database according to given cycle. Patient vital sign data and log files are recorded. The product has the function of backing up log regularly and providing log query interface.

\section{Design of Node Software}

There are three nodes with different functions in monitoring network, namely ZigBee coordinator, ZigBee router and sensor node. TI Company's Z-Stack protocol stack is adopted in system underlying layer on all nodes. Network parameter configuration of all nodes is the same. After coordinator is started, all appointed channels are firstly scanned, the best channels are selected for composing network. After router and sensor node are started, appointed channel is scanned, which is added into network according to value.

\section{Design of Monitoring Center Software}

In the paper, QT4.6.0 is used for developing monitoring center software system. QT belongs to a cross-platform $\mathrm{C}++$ graphical user interface library. It has good cross-platform performance, which can be operated on embedded platform. Therefore, monitoring center software designed in the paper can well support multi-platform operation \# application software of the monitoring system is divided into several functional modules according to demand analysis on monitoring network, including parameter setting module (system setting, patient information setting, network parameter setting, etc.), real-time task processing module (real-time data management, real-time data display, real-time data processing and data abnormal alarm), and data storage processing module (for realizing data playback, data analysis, digital data transmission, printing output, etc.), Determined tasks should be respectively completed in all modules. Modules are mutually independent relatively, which are mutually connected through systematic framework agreement.

The system software is divided into three parts: data acquisition and processing, data transmission and reception, and data display. Wherein some codes of data acquisition and processing are operated in sending end, which is mainly used for collecting data as shown in figure 3. Data transmission and reception are operated in receiving end (i.e. gateway node), which is mainly used for data transmission.

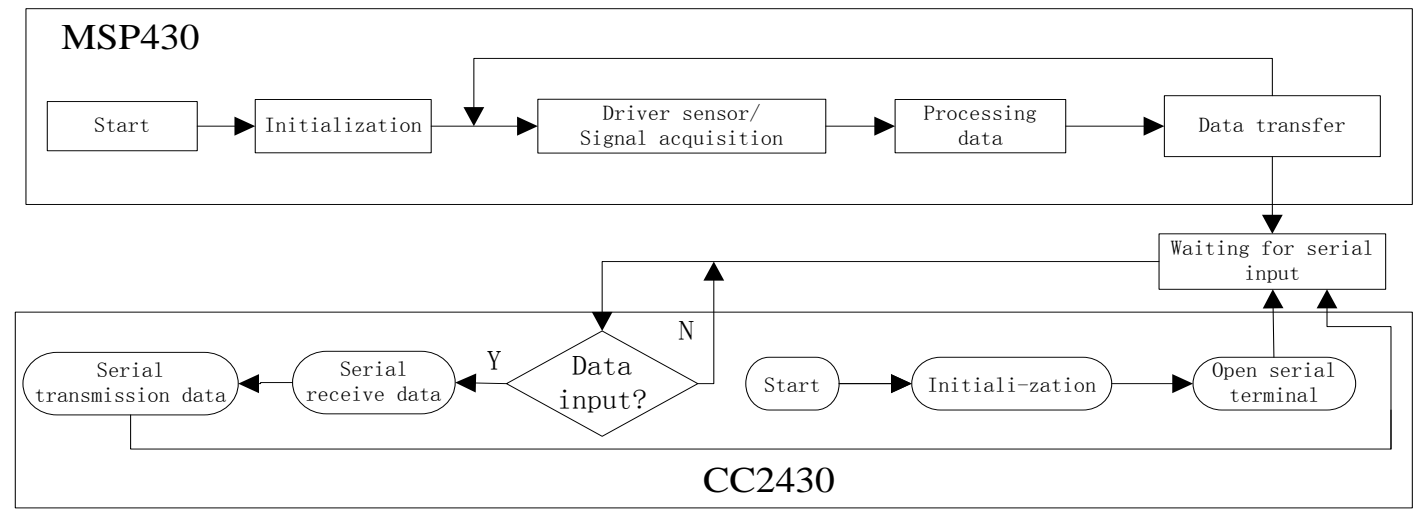

Fig.3. Wireless Sensor Node Data Flow Diagram

Codes in data show part are operated in PC machine, which are mainly used for waveform display and further processing of data received by PC machine as shown in figure 4. After wireless sensor nodes and gateway nodes are established, and point-to-point communication can be realized by ZigBee protocol then. 


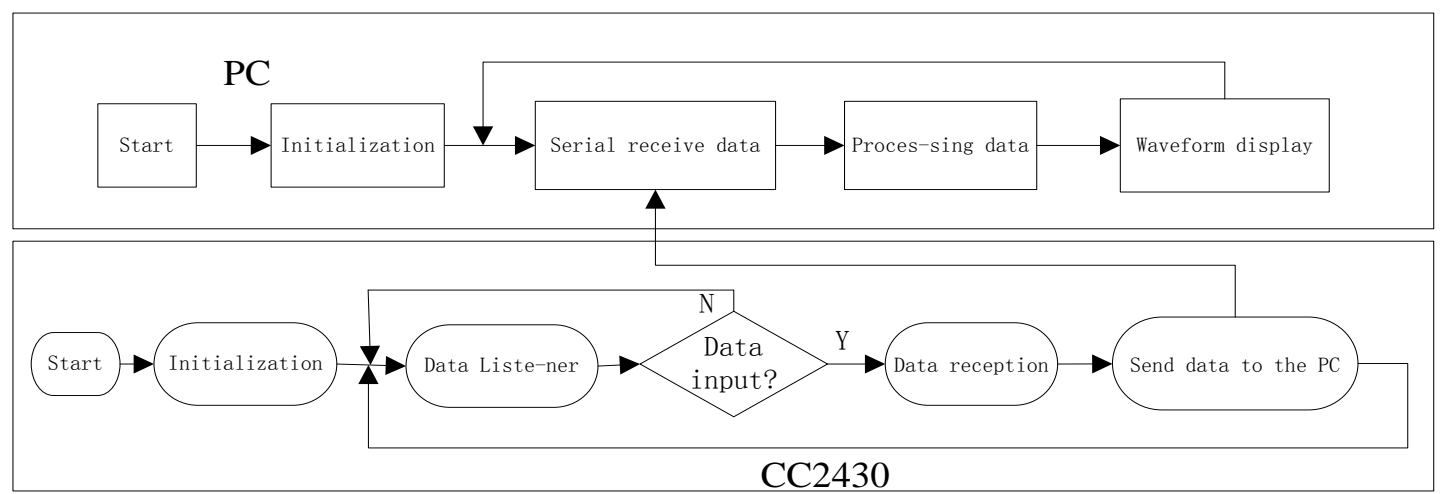

Fig.4. Gateway Mode Data Flow Diagram

\section{Conclusion}

Monitoring system designed in the paper aims at transmitting information between medical sensor and monitoring control instrument by high frequency wireless multi-channel data transmission mode. Wiring between monitoring equipment and medical sensor is reduced. The monitored patients can enjoy more free activity space. When more accurate measurement indicators are obtained, rushing tiredness of patients between family and hospital can be avoided. Medical monitoring system based on wireless sensor network is development trend for domestic and foreign medical equipment. In the paper, wireless monitoring system is constructed with ZigBee technology as basis in the paper. It has advantages of low power, small size, low network operation cost, etc. However, there are many problems that should be studied at the same time, such as information security, node localization, fault tolerance mechanism, etc. Wireless sensor network is characterized by low cost, high flexibility, etc., thereby it has wide application prospect in hospital and community medical monitoring services with development of wireless communication technology.

\section{Acknowledgement}

In this paper, the research was sponsored by the Qiqihar Municipal Bureau of Science and Technology (Project No. GY2D2013012).

\section{References}

[1] Daping Dong, Xiaoying Tang, Weifeng Liu. Research on Applications of Wireless Sensor Networks On Medical Monitoring[J]. Life Science Instruments.2008,6:26-28.

[2] Jumei Zhang, WU Xiaoming Wu. Development of Real-time Monitoring System Based on ZigBee in ICU Wards[J]. Chinese Medical Equipment Journal.2009,30(7):28-30.

[3] Houjun Xiao, Weidong Hao, Yiming Liu. Design of the Wireless Medical Monitoring System Based on ZigBee[J]. Microcomputer Information.2012,28(1):74-76.

[4] EGAN D. The emergence of ZigBee in building automation and industrial control[J]. Computing\&Control Engineering Journal.2005,16(2):14-19.

[5] Huiqian Luo, Yi Ren, Daosheng Shi. A Wireless Health Care System Based on ZigBee Technology[J]. China Water Transport.2007,5(4):148-149. 